\title{
Les sans-abri d'Old Delhi : insertion urbaine et stratégies économiques
}

\section{Véronique Dupont}

\section{(2) OpenEdition}

1 Journals

\section{Édition électronique}

URL : http://journals.openedition.org/conflits/169

DOI : $10.4000 /$ conflits. 169

ISSN : $1777-5345$

Éditeur :

CCLS - Centre d'études sur les conflits lilberté et sécurité, L'Harmattan

\section{Édition imprimée}

Date de publication : 1 septembre 1999

ISBN : 2-7384-8616-9

ISSN : 1157-996X

\section{Référence électronique}

Véronique Dupont, "Les sans-abri d'Old Delhi : insertion urbaine et stratégies économiques », Cultures \& Conflits [En ligne], 35 I automne 1999, mis en ligne le 16 mars 2006, consulté le 30 mars 2021. URL: http://journals.openedition.org/conflits/169 ; DOl : https://doi.org/10.4000/conflits.169

Ce document a été généré automatiquement le 30 mars 2021.

Creative Commons License 


\title{
Les sans-abri d'old Delhi : insertion urbaine et stratégies économiques
}

\author{
Véronique Dupont
}

L'absence de toit apparait à première vue comme une des facettes les plus révoltantes de la pauvreté, et les sans-abri comme les «pauvres parmi les pauvres »" Ainsi, dans les grandes métropoles indiennes, la présence de longs alignements de personnes dormant la nuit sur les trottoirs est d'abord appréhendée comme la manifestation de situations de pauvreté aiguë, traduisant des formes d'exclusion du logement et des services de base. A un niveau d'interprétation micro-social, ces sans-abri ont été décrits comme « les victimes malheureuses de divers types de crises physiques et sociales affectant les sociétés rurales et urbaines » en Inde ${ }^{2}$. Au niveau macro-social, la présence des sansabri dans les grandes villes et leur nombre croissant ont été analysés comme «un produit inévitable du processus d'urbanisation " ${ }^{3}$, la conséquence de l'industrialisation et $\mathrm{du}$ développement économique qui induisent la migration des pauvres des campagnes vers les villes, avec une pression résultante sur les terrains et sur le logement ${ }^{4}$. Sans nier ni minimiser cette dimension de la situation des sans-abri, il nous semble important de souligner que, contrairement aux situations généralement observées dans les villes françaises ou des Etats Unis ${ }^{5}$, dans le contexte indien, à Delhi comme à Bombay ou Calcutta, cette exclusion du logement en ville n'a pas pour corollaire inéluctable perte du lien social et marginalisation économique. Pour comprendre les différentes facettes de la persistance des situations de sans-abri, il est nécessaire de ne pas considérer les «squatters des rues" uniquement comme des victimes d'un processus de paupérisation, mais également comme des agents dynamiques capables de mettre en œuvre des stratégies économiques, ou pour le moins des tactiques de survie adaptatives, et de trouver des réponses appropriées à un environnement urbain spécifique. C'est dans cette perspective que nous analyserons ici les pratiques résidentielles et économiques de la population des sans-logis dans old Delhi, le centre historique de la capitale indienne ${ }^{6}$. Une telle investigation nous permettra également d'aborder sous différents angles la question de la place des sansabri dans la société indienne: l'intégration dans les structures familiales et communautaires ou l'individualisation, la marginalisation sociale et l'anomie; 
l'insertion dans le marché du travail et le rôle dans les économies urbaines et rurales; l'exclusion du logement et la mise en œuvre de stratégies résidentielles et économiques adaptatives; la place disputée dans l'espace urbain, les droits des sans-logis, et, finalement, la place dans la société civile. Les résultats discutés dans cet article s'appuient sur les données de nos propres enquêtes socio-économiques; une brève présentation de celles-ci complétée par une revue des données secondaires disponibles, ainsi qu'une réflexion préalable incontournable sur le concept de sans-abri, feront l'objet d'une section préliminaire. Le concept de sans-abri Bien que les sans-abri soient souvent qualifiés de plus pauvres parmi les pauvres - «the poorest among the poor " ${ }^{\text {, }}$ à part quelques exceptions ${ }^{8}$, la question des sans-logis est en général négligée dans la littérature abondante consacrée à la pauvreté urbaine et aux problèmes liés à l'urbanisation en Inde ${ }^{9}$; de même les enquêtes sur cette sous-population sont relativement peu nombreuses ${ }^{10}$, comparées à l'abondance des travaux sur les habitants des bidonvilles dans les villes indiennes. On note en particulier un manque flagrant d'études spécifiques et d'enquêtes de terrain sur la population des sans-logis à Delhi ${ }^{11}$. Conséquence directe de cette carence, on dispose de peu d'informations précises sur ce segment de la population urbaine, à commencer sur son effectif. Le recensement de 1991 dénombrait 50000 personnes sans-logis dans l'agglomération urbaine de Delhi, chiffre qui, de toute évidence, sous-estime fortement la réalité, la raison tenant sans doute aux conditions mal appropriées de repérage et d'énumération des sans-logis pendant les opérations du recensement général de la population. Selon d'autres estimations plus réalistes ${ }^{12}$, l'effectif des sans-logis à Delhi représenterait à environ $1 \%$ de la population urbaine totale, ce qui correspondrait à environ 100000 personnes au milieu des années quatre-vingt dix (pour une agglomération urbaine de près de dix millions d'habitants à cette date). Les concentrations les plus importantes et les plus visibles de personnes sans-abri se trouvent dans la vieille ville de Delhi et ses quartiers adjacents, où la Municipalité a - logiquement - ouvert dix de ses dix-huit asiles de nuit, représentant les trois quarts du total des 4500 places mises à la disposition des sansabri dans toute l'agglomération urbaine. Comme nous l'exposerons dans la section suivante, les caractéristiques morphologiques et économiques du centre historique de la capitale contribuent à l'attraction spécifique exercée sur une population flottante de travailleurs migrants sans-abri. Ce contexte particulier explique le choix de centrer nos enquêtes sur les sans-abri dans Old Delhi. La terminologie et les définitions utilisées dans les travaux sur les sans-logis à Delhi, ou dans d'autres villes indiennes, sont loin d'être homogènes. Ainsi les termes de houseless (sans-logis), homeless (sans-domicile, sans foyer), roofless (sans-toit), shelterless (sans-abri), pavement dwellers (squatters des trottoirs) ne recouvrent pas toujours les mêmes groupes de population, un même terme peut en outre correspondre à des définitions différentes selon les études de cas considérées. Par exemple, les travaux consacrées aux personnes vivant dans la rue (pavement dwellers) se limitent dans certains cas aux seules personnes dépourvues de tout $\mathrm{abri}^{13}$, alors que d'autres incluent également celles qui se protègent par des abris de fortune ou constructions précaires érigées sur les trottoirs ${ }^{14}$. Dans certaines études sur Delhi, les personnes dormant la nuit dans les asiles ouverts par la Municipalité sont également inclues dans la population des sans-logis. Le recensement indien utilise la notion de «houseless population» (population sans-logis), définie comme les personnes ne vivant pas dans un logement au sens du recensement, c'est à dire dans une structure pourvue d'un toit. Ainsi, les agents recenseurs ont pour instructions de noter tous les endroits possibles où les personnes sans-logis sont susceptibles de vivre, 
tels : le long des routes, sur les trottoirs, dans les tuyaux des grosses canalisations, sous les cages d'escaliers, en plein air, près des temples, sur les quais, etc. ${ }^{15}$. En fait, des difficultés similaires d'estimations fiables des effectifs et de définition de la population des sans-logis se retrouvent dans les enquêtes conduites dans les villes américaines et européennes Firdion (J. M.), Marpsat (M.), Bozon (M.), « Est-il légitime de mener des enquêtes statistiques auprès des sans-domicile ? Une question éthique et scientifique ", Revue Française des Affaires Sociales, 49ème année, n² 2-3, 1995, pp. 29-51; Firdion (J. M.), Marpsat (M.), «La statistique des sans-domicile aux Etats-Unis », Courrier des Statistiques, n 71-72 - Déc. 1994, pp. 43-51; Wright (J. D.), Robin (B.), «Les sansdomicile aux Etats Unis ", Sociétés Contemporaines, n 30, 1998, pp. 35-66. ]]. Comme le note justement Bienveniste à propos du cas français: «Etre sans abri n'est pas une caractéristique définissant une sous-population mais bien plutôt une situation commune à des populations hétérogènes à un moment donné de leur histoire " ${ }^{16}$. Cette remarque permet de souligner deux caractéristiques majeures de la population des sans-logis: son hétérogénéité et sa mobilité. La population spécifique soumise aux enquêtes est limitée aux personnes dépourvues, à Delhi, de tout logement ou abri personnel et permanent, si précaire soit-il, et dormant dans les espaces publics ou dans les asiles de nuit de la municipalité. Les personnes érigeant sur les trottoirs des abris de fortune ou constructions précaires ne sont pas inclues dans notre étude; de fait dans l'enceinte de la vieille ville où nous avons concentré nos enquêtes, de telles situations ne sont pas observables. Nous utiliserons ici de manière indifférenciée les termes de sans-abri ou sans-logis pour désigner la population enquêtée. En revanche le terme de sans domicile, en particulier l'expression consacrée en France de Sans Domicile Fixe, qui correspondrait à l'équivalent anglais de homeless, terme utilisé couramment dans le contexte des Etats-Unis où il traduit une certaine réalité sociale ${ }^{17}$, ne nous semble pas approprié au contexte des villes indiennes. En effet, la situation de sans-logis dans Old Delhi n'implique pas nécessairement une absence de «foyer» ou de domicile de base. Le concept de famille doit en effet dépasser le concept de ménage, ou de foyer, rattaché à une unité résidentielle unique, de manière à pouvoir intégrer des segments familiaux spatialement éclatés mais reliés entre eux par des liens de solidarités économiques et affectives. Ainsi, l'identification de la population des sans-logis fait ici référence à une situation de fait (l'absence d'abri) à un moment donné, dans un endroit donné (pendant la période d'observation, à Old Delhi). Mais la situation observée à Delhi n'est pas nécessairement un état permanent et peut être compatible avec l'existence d'un domicile et d'un foyer dans un autre lieu, en particulier dans le village d'origine. Les enquêtes socio-économiques à la base de cette étude ont associé deux types d'observation: une enquête statistique conduite de janvier à mars 1996, couvrant un échantillon de 248 personnes sélectionnées par sondage aréolaire dans les principales concentrations extérieures de sans-logis dans l'enceinte fortifiée de la vieille ville et dans les six asiles de nuit ouverts par la Municipalité dans cette même zone; des entretiens approfondis conduits simultanément auprès d'un sous-échantillon de 36 individus tirés de manière aléatoire ${ }^{18}$. Old Delhi : transformations de l'espace urbain et population sans-logis Déconcentration de la population et intensification des activités économiques Old Delhi, le centre historique de la capitale construit par les Moghols au 17ème siècle, se caractérise par des densités de population extrêmement élevées, combinées à une très forte concentration de commerces et de petites entreprises industrielles. Un double mouvement marque la dynamique de ce vieux centre. Un dépeuplement résidentiel est d'une part observable depuis la décennie 1961-71 dans 
certains secteurs ${ }^{19}$ et cette tendance s'est depuis confirmée et généralisée, les densités de population restant toutefois extrêmement élevées (616 personnes à l'hectare en 1991 dans l'enceinte fortifiée qui couvre 600 hectares). D'autre part, les établissements commerciaux et les ateliers industriels ont proliféré. Le nombre d'établissements commerciaux enregistrés s'est ainsi accru de 700\% en vingt ans, de 22000 unités en 1961 à 155000 en $1981^{20}$. En outre, comme souligné justement par Mehra ${ }^{21}$, ces statistiques officielles sous-estiment la croissance des activités économiques, dans la mesure où les activités informelles échappent à ce recensement. Transformations de la morphologie urbaine et recomposition sociale La sur-utilisation du bâti ancien dans la ville fortifiée - à la fois en termes d'usage résidentiel et économique - a contribué à la dégradation du parc immobilier. Le cycle de détérioration à l'œuvre est aussi un effet pervers de la loi de blocage des loyers de 1958 qui, en maintenant ces derniers à un très bas niveau, engendre des revenus insuffisants pour les propriétaires, qui rechignent de fait à engager des dépenses d'entretien de leur bâtiment ${ }^{22}$. L'ensemble de la ville fortifiée a d'ailleurs été déclaré zone d'habitat insalubre (« notified slum ») depuis 1956. La transformation de l'usage du bâti s'accompagne d'une certaine recomposition sociale de la population résidente. D'un côté, on observe des départs de familles de commerçants, négociants et entrepreneurs propriétaires à la recherche de meilleures conditions de logement dans des quartiers moins congestionnés - mais qui conservent leur maison et maintiennent leurs activités dans la vieille ville. Ce sont ainsi les sections les plus aisées qui partent, laissant sur place les groupes aux revenus plus faibles, en particulier les locataires qui ne seraient pas en mesure de trouver des logements en location à des taux si bas dans d'autres localités du fait de la loi de blocage des loyers dont ils continuent à bénéficier dans la vieille ville ${ }^{23}$. Par ailleurs, la prolifération des activités commerciales et artisanales ainsi que des services associés, pourvoyeurs de nombreux emplois informels, a attiré une population flottante de travailleurs migrants masculins non qualifiés, en particulier en provenance des campagnes pauvres du nord de l'Inde. L'insertion résidentielle de ces migrants reste des plus précaires : nombreux sont ceux que l'on retrouve la nuit dormant sur leur lieu de travail, dans les asiles de nuit municipaux, sous les arcades du bazar, le long des trottoirs, sur les passerelles piétonnes et les emplacements de marché, dans les parcs et autres espaces publics. La localisation dans la vieille ville ou aux portes de celle-ci des deux principales gares ferroviaires de la capitale et de la principale gare routière interrégionale joue un rôle important, non seulement en contribuant au dynamisme économique général de la zone et à la création d'emplois, mais aussi en fixant les migrants sans-abri dans ces quartiers : à leur arrivée, ces derniers sont en contact direct avec (ou aisément dirigés vers) les principaux lieux d'embauche pour les travailleurs non qualifiés, et ils peuvent facilement trouver dans le voisinage des emplacements de couchage extérieurs et des asiles de nuit. Services développés pour les sans-logis Une série de services spécialement orientés vers les besoins de cette population sans-logis a aussi vu le jour. Les pouvoirs publics, reconnaissant l'ampleur du phénomène des sans-abri, ont commencé à ouvrir des asiles de nuit au début des années soixante. Dans ces asiles gérés par la municipalité de Delhi, les personnes sanslogis trouvent, contre un modique droit d'entrée de trois roupies la nuitée (en 1996), un abri pour la nuit avec une couverture et un tapis de sol, et l'accès à des sanitaires. Certains entrepreneurs privés ont visualisé la situation de sans-abri de si nombreuses personnes comme un créneau à occuper : ils louent des couettes ou même des lits à la nuitée, aux taux moyens de cinq et quinze roupies respectivement. La plupart d'entre 
eux offrent également des emplacements de couchages « aménagés » à leurs clients : ils occupent la nuit certaines sections des trottoirs, en particulier ceux protégés par l'encorbellement des étages des bâtiments, ou encore des passerelles piétonnes, les emplacements de marché et autres espaces publics extérieurs; des bâches en plastique sont disposées sur le sol en guise de protection, ou bien de lits. Pour les zones de couchages entièrement en plein air, des bâches en plastique sont également installées de manière à protéger les dormeurs en cas de pluie. Ces facilités de couchage sont particulièrement demandées en hiver, lorsque la température nocturne peut descendre jusqu'à trois degrés centigrades. Une proportion importante des sans-logis n'ont toutefois pas recours à ces solutions de couchage payantes, et étalent leur propre couverture sur les trottoirs et autres espaces publics libres d'accès - ils doivent compter néanmoins sur les opérations de police éventuelles ${ }^{24}$. Parmi d'autres services annexes qui se sont développés pour répondre aux besoins spécifiques de la population des sans-abri, on peut signaler des vendeurs de plats cuisinés qui viennent le soir installer pour quelques heures leur réchaud à même le trottoir, ou encore les nombreux préparateurs-vendeurs de thé opérant dans ou près des zones de couchage. Les sanslogis : des marginaux sociaux ou des migrants intégrés? Dans un premier temps, nous essaierons d'évaluer le degré de marginalité ou d'intégration sociale des migrants sanslogis, avec une série de questionnements interdépendants. Dans quelle mesure la situation actuelle de sans-abri est-elle le résultat d'une crise, d'une rupture d'avec les systèmes traditionnels de solidarité et la conséquence d'un processus d'individualisation, menant à des formes d'anomie dans l'environnement de la grande ville? La migration à Delhi s'intègre-t-elle dans des stratégies familiales enracinées dans le milieu d'origine? Quelle est alors la pertinence de l'institution sociale de base, la famille, et la signification des attaches rurales des migrants pour comprendre les trajectoires et les pratiques économiques et résidentielles actuelles des sans-logis? Au préalable, l'établissement du profil socio-démographique et de l'histoire migratoire des sans-logis nous aideront à dresser un premier portrait de cette population en nous livrant également des indices de son intégration sociale. Profil socio-démographique Premier trait remarquable, la population des sans-abri d'Old Delhi est constituée presque exclusivement d'hommes vivant seuls ${ }^{25}$, alors que certains quartiers de la capitale "accueillent" également des familles sans-logis. Cette prédominance masculine est aussi une caractéristique de la population des sans-logis de Delhi comparée à celle des autres grandes métropoles indiennes. Ainsi, selon les données du recensement de 1981 (les tableaux correspondant du recensement de 1991 n'ayant pas été publiés) la population des sans-logis dans les quatre plus grandes métropoles comprenait : 187 femmes pour 1000 hommes sur l'ensemble de l'agglomération urbaine de Delhi contre 453 pour 1000 à Calcutta, 278 pour 1000 à Bombay et 955 pour 1000 à Madras. La taille moyenne des ménages sans-logis illustre à nouveau la position particulière de la capitale : 1,9 à Delhi dans son ensemble contre 4,8 à Calcutta, 2,0 à Bombay et 4,1 à Madras. La quasi absence de familles vivant sur les trottoirs d'old Delhi se reflète dans la structure par âge de la population des sans-abri. Si, d'un point de vue humanitaire, la présence d'enfants vivant dans la rue, livrés à eux-mêmes, souligne une des dimensions les plus alarmantes de la condition des sans-abri, en termes démographiques les enfants demeurent un groupe très minoritaire dans l'ensemble des sans-logis (dans l'échantillon, moins de $5 \%$ des enquêtés ont moins de 15 ans). La majorité des sans-abri de la vieille ville sont de jeunes adultes : $54 \%$ d'entre eux sont âgés de 15 à 29 ans. Plus significatif du point de vue de leur intégration sociale : le 
statut matrimonial des sans-abri. Ainsi, 78 \% des enquêtés âgés de 15 à 29 ans n'ont jamais été mariés, et $43 \%$ parmi ceux âgés de 30 ans et plus. Afin de mieux apprécier la spécificité de la population des sans-logis, ces chiffres peuvent être comparés aux pourcentages correspondant d'hommes jamais mariés sur l'ensemble de la population urbaine de Delhi, soit, respectivement : $62 \%$ et $3 \%$ selon le recensement de 1991 . Le pourcentage remarquablement élevé de célibataires à des âges relativement avancés peut être considéré, dans le contexte de la société indienne, comme l'indice d'un certain degré de marginalisation sociale parmi une section des sans-logis, la manifestation d'un processus d'individualisation, choisi ou subi. Les circonstances de la migration vers Delhi et plus généralement les histoires de vie nous fourniront quelques éléments explicatifs. Le pourcentage de castes d'ex-intouchables parmi les sans-abri permet d'évaluer dans quelle mesure cette population est issue des couches les plus défavorisées de la société. Parmi les sans-logis enquêtés dans Old Delhi, 13\% ont déclaré appartenir au groupe des castes ou tribus répertoriées parmi les plus défavorisées (Scheduled Castes \& Tribes). Compte tenu des erreurs d'échantillonnage, ceci représente une proportion très proche de celle observée dans la population masculine résidant en 1991 dans l'enceinte de la vieille ville, soit 11\%, et nettement inférieure à celle observée dans la population masculine de l'ensemble de l'agglomération urbaine, soit $19 \%$. Les données du recensement ne permettent pas de comparaison pour les autres castes et communautés, on notera cependant que la majorité des sans-logis enquêtés dans Old Delhi appartiennent à des castes ou communautés considérées de rangs supérieurs (56\% d'entre eux), la part restante (31\%) correspondant aux groupes classés comme économiquement en retard (Other Backward Classes). La proportion d'analphabètes procure un autre éclairage des handicaps socio-économiques. De ce point de vue, les sans-abri d'old Delhi apparaissent nettement comme un segment défavorisé de la population, comptant $38 \%$ d'analphabètes, alors que cette proportion n'est que de $24 \%$ dans la population masculine résidant dans l'enceinte de la vieille ville en 1991, et $18 \%$ dans la population masculine de l'ensemble de l'agglomération urbaine de Delhi ${ }^{26}$. Histoire migratoire La migration est une expérience commune partagée par la presque totalité des sans-logis d'old Delhi (96\% des enquêtés). La grande majorité de ces migrants (61\%) sont arrivés directement de leur village ou ville natale dans la capitale, près des trois quarts sont d'origine rurale, et $69 \%$ en provenance de l'Uttar Pradesh et du Bihar, deux Etats pauvres et densément peuplés du nord de l'Inde. L'examen des raisons à l'origine du départ du lieu d'origine (ou du foyer familial) et de la migration vers Delhi (ou de l'arrivée sur le trottoir) révèle des situations très contrastées. Un premier point marquant est l'impact des tensions familiales, disputes et mauvais traitements ${ }^{27}$. Les cas d'enfants battus par un père ivre ou maltraités par leur belle-mère, de querelles à propos du partage de la propriété familiale après la mort du père, de disputes avec l'épouse, le frère ou tout autre parent,... etc., sont fréquemment signalés. Quand la migration vers Delhi correspond à un départ du milieu d'origine dans des circonstances de crise familiale, la préoccupation principale pour le migrant est de fuir une situation devenue insupportable. Dans de tels cas, il n'est pas surprenant que l'arrivée à Delhi n'ait pas été préparée, que le choix de la destination n'ait pas été envisagé au préalable, laissé parfois au hasard du premier train en partance. Si la fuite $\mathrm{du}$ foyer originel est suivie par une rupture de tous les liens avec la famille, et si le migrant est encore célibataire au moment de son départ, il aura toutes les chances de le rester, ne pouvant plus bénéficier de l'appui de sa famille pour arranger son mariage selon la coutume en vigueur. Plus conforme au schéma général de migrations 
prévisibles pour une grande métropole, une large majorité des migrants sans-logis enquêtés (66\%) ont choisi de venir à Delhi pour des raisons économiques, liées à des perspectives d'emploi plus favorables dans la capitale. Cette catégorie inclut de nombreux migrants originaires de zones rurales ou de petites villes, et dont les revenus dans le lieu d'origine n'étaient pas suffisants pour entretenir leur famille, mais aussi des jeunes gens désireux de gagner leur vie de manière autonome, en dehors de l'agriculture familiale. Conditions de l'insertion en ville Ainsi que nous l'avons exposé dans un précédent article ${ }^{28}$, l'information préalable sur les conditions de vie et les possibilités d'emploi à Delhi ainsi que l'aide reçue à l'arrivée en ville mettent en évidence le rôle du réseau des apparentés et originaires du même village ou de la même région pour la circulation de l'information et l'insertion en ville des migrants sanslogis. Le rôle de ce réseau est également observé dans le cas des migrants dont l'insertion résidentielle n'est pas aussi précaire ${ }^{29}$. Outre l'attraction propre à une grande métropole et à ses perspectives d'emplois multiples, le choix de Delhi est souvent influencé par la présence de parents, d'alliés ou de co-villageois travaillant déjà dans la capitale, et transmettant des informations sur les possibilités d'emploi. Parmi ceux qui reconnaissent avoir été aidés dans leur première insertion en ville (soit les deux tiers des personnes interviewées), pour trouver un travail ou un endroit pour dormir, le réseau des parents et des co-villageois est abordé dans la majorité des cas, montrant que la condition actuelle de sans-abri ne signifie pas nécessairement que ces migrants opèrent dans un vide familial et social. Au cours du séjour en ville, le lieu de travail et la communauté des personnes travaillant dans la même branche d'activité procurent le réseau de sociabilité principal des personnes sans-logis. Pour les plus jeunes et les plus âgés des sans-logis, le groupe d'âge est aussi un groupe de référence privilégié pour trouver des amis et dormir dans les mêmes lieux. Les personnes originaires du même village ou de la même région restent dans certains cas un groupe de socialisation significatif. Dans cette perspective, un type intéressant de migration est à signaler parmi les sans-logis : les filières migratoires de tradition familiale ou même villageoise. On peut ainsi trouver des groupes de travailleurs migrants saisonniers en provenance d'un même village, dormant sur le même coin de trottoir bien que de castes différentes, cuisinant parfois ensemble, retournant ensemble au village pour les fêtes principales, s'organisant pour envoyer régulièrement l'un d'entre eux rapporter les économies de tous et les redistribuer à leurs familles respectives. Une vie communautaire a pu être de la sorte reconstituée sur les trottoirs de la capitale, fondée sur l'appartenance au même lieu d'origine, et ce lien transcende les différences de caste, du moins pendant le séjour à Delhi, dominé par des préoccupations liées au travail. Toutefois une proportion notable de sans-logis interrogés dans old Delhi déclarent n'avoir de contact avec personne sur leur lieu de couchage, la majorité d'entre eux prenant leur dîner seuls. Les tactiques d'évitement de certains sans-abri dans les lieux de couchage révèlent aussi la persistance des préjugés de castes et leur méfiance envers les communautés religieuses autres que la leur. Relations maintenues avec le milieu d'origine La plupart des personnes sans-abri enquêtées dans la vieille ville ont des membres de leur famille dans leur localité d'origine et la majorité d'entre elles s'y rendent plus ou moins régulièrement pour des visites - au moins une fois pendant les deux dernières années - ou ont l'intention de le faire dans le cas des migrants nouvellement arrivés. En outre, environ la moitié des sans-logis qui ont encore de la famille dans leur village (ou ville) natal(e) leur procure une aide financière, souvent complétée par l'achat de vêtements ou produits ménagers au moment des 
visites. L'attachement à la famille et au lieu d'origine est encore révélé par les projets futurs de retour au "village» (dans les prochaines années ou beaucoup plus tard), souhait partagé par la majorité des personnes interrogés. Sur la base de cette première investigation, on peut dresser une typologie des migrants sans-logis vivant seuls dans la vieille ville en fonction de leur degré d'enracinement au lieu d'origine. En particulier deux types distincts et diamétralement opposés de situations peuvent être identifiés, avec tout l'éventail des situations intermédiaires. Le premier, correspondant au plus haut degré d'intégration familiale et d'attachement au village, regroupe les migrants saisonniers venant chaque année travailler à Delhi pour quelques mois, habituellement pendant la basse saison agricole, et qui soutiennent directement leur famille dans leur lieu d'origine. On trouve proche de ce groupe, les migrants mariés dont l'épouse et les enfants (s'ils en ont) restent au "village » avec le reste de la famille élargie, et qui envoient régulièrement des mandats, ainsi que les migrants non mariés qui contribuent au revenu familial ; ces deux groupes visitant régulièrement leur famille. Ces migrantsremetteurs exemplifient un principe de solidarité familiale transcendant l'unité résidentielle. Ceci est une caractéristique courante du procès migratoire, en particulier des migrations des campagnes vers les villes, en Inde comme dans d'autres pays en développement. Le point qui mérite d'être souligné ici est le fait que la condition de sans-abri du migrant en ville ne l'empêche pas d'exercer cette solidarité ; en fait, c'est précisément cette condition qui lui permet de soutenir financièrement sa famille. Le deuxième, correspondant au degré le plus bas de l'attachement à la communauté d'origine, regroupe les personnes sans-logis, engagées dans un processus d'individualisation et d'anomie, qui ont fui leur foyer - en particulier des enfants - à la suite de tensions familiales aiguës, souvent accompagnées de violences, et qui ont ensuite coupé tous les liens avec leur famille et leur lieu d'origine. Etant donné les circonstances du départ du foyer, ces migrants ou « réfugiés » ne peuvent pas compter sur les réseaux familiaux et villageois pour leur insertion dans une nouvelle ville. On pourrait rattacher à ce dernier groupe certains migrants issus de hautes castes qui ont dû quitter leur lieu d'origine en raison d'une situation économique très tendue ou suite à une crise financière soudaine. S'ils ont choisi Delhi comme lieu de migration, c'est, notamment, en raison du large éventail de possibilités d'emploi offertes dans une grande métropole. Mais ce choix s'explique avant tout pour l'anonymat que procure un tel environnement urbain, leur permettant ainsi d'exercer des activités, en particulier des métiers manuels, que leur statut social ne leur permettrait pas d'exercer dans leur milieu d'origine. Loin du village ou de la ville natale et loin de la communauté de base, c'est-à-dire loin du milieu social d'origine, l'éventail des métiers acceptables s'élargit pour le migrant. Toutefois, le coût psychologique de telles stratégies de survie peut s'avérer élevé. Personne dans le village (ou ville) d'origine ne devant connaître leurs conditions de vie et de travail à Delhi, ces migrants ne donnent aucune adresse, n'échangent aucune nouvelle par lettre ou messagers, évitent de rencontrer des personnes de leur localité d'origine ; en outre, ils semblent accablés par la honte d'avoir à exercer un métier peu « respectable » et par leur condition de sans-abri. Ce sentiment de honte a incité certains d'entre eux à couper tout lien avec leur famille et lieu d'origine, et à Delhi, ils réduisent également leurs relations avec les autres sans-abri, aggravant ainsi leur isolement.

La rupture avec la famille, institution traditionnelle de base, ne s'applique toutefois qu'à une section limitée des sans-logis. Nous retiendrons plutôt, en conclusion de cette première analyse, que, vivre seul et sans-abri à Delhi n'implique pas nécessairement 
vide familial et marginalité sociale. Pour la majorité des migrants sans-abri interrogés dans la vieille ville, le groupe familial et la localité d'origine restent leur pôle de référence. En outre, la situation de sans-abri des migrants dans la capitale ne s'oppose pas au maintien d'une solidarité familiale, au contraire - comme nous le développerons dans une autre section - elle en est souvent une condition nécessaire.

Insertion dans le marché du travail urbain et lien avec l'économie rurale L'examen de la condition économique des sans-abri dans old Delhi nous permettra d'aborder la question de leur insertion dans le marché du travail urbain et des liens avec l'économie rurale. Cette remise en cause de la marginalité économique de la population des sanslogis nous conduira à une réflexion plus générale sur le concept de pauvreté.

Tableau 1. Répartition de la population des sans-logis dans Old Delhi par activité économique en $1996^{30}$.

Activité économique Activité principale Autres activités Toutes activités $\mathrm{N}^{\circ} \% \mathrm{~N}^{\circ} \% \mathrm{~N}^{\circ} \%$ Employés de bureau 2 0.8 - - 20.6 Vendeurs, aides chez les commerçants 104.111 .211 3.4 Cuisiniers, serveurs et autres aides 5422.24656 .110030 .8 Employés domestiques, barbiers, et autres services personnels 20.822 .441 .2 Ouvriers de la production, y compris mécaniciens et réparateurs 166.622 .4185 .5 Ouvriers du bâtiment 229.178 .5 298.9 Portefaix 218.61012 .2319 .5 Tireurs de charrettes à bras 5823.978 .56520 .0 Tireurs de cyclo-rickshaws 4819.867 .35416 .6 Conducteurs de véhicules à moteur 10.4 11.220 .6 Collecteurs de déchets pour leur recyclage 52.1 - - 51.5 Mendiants 31.2 - - 3 0.9 Autres activités (musicien) 10.4 - - 10.3 Total 243100.082100 .0325100

Tableau 2. Répartition de la population des sans-logis dans Old Delhi par catégorie industrielle en 1996 et comparaison avec la population urbaine de Delhi au recensement de $1991^{31}$.

Catégorie industrielle population sans-logis old Delhi 1996 Population masculine old Delhi 1991 Population masculine Delhi 1991 (1) (2) (3) Agriculture, élevage, mines, carrières 0.00 .61 .3 Industries manufacturières 6.232 .026 .4 Construction 9.02 .87 .9 Commerce, restaurants, hôtels 28.438 .326 .4 Transport, communication 51.97 .98 .8 Services communautaires, sociaux et personnels 4.5 18.329.2 Total 100.0 100.0 100.0

Participation à la force de travail urbaine et éventail des activités économiques Les sans-abri d'old Delhi font partie intégrante de la force de travail urbaine. La proportion des enquêtés n'ayant reporté aucune activité génératrice de revenus reste marginale (2\% de l'échantillon) ${ }^{32}$, ce sont essentiellement des migrants très récents (arrivés il y a moins d'un mois) et encore à la recherche d'un emploi. Ce constat sur l'intégration des sans-abri dans le marché du travail de la capitale est en accord avec les résultats des études précédentes sur les sans-logis à Delhi ${ }^{33}$. Les situations rencontrées au regard de l'emploi et des revenus restent toutefois très variées et disparates. Les différents types d'activités exercées par les sans-logis reflètent clairement les fonctions économiques spécifiques d'old Delhi ${ }^{34}$. On remarque tout d'abord le large groupe des tireurs de charrettes à bras transportant des marchandises dans les marchés de gros de la vieille ville ( $24 \%$ des enquêtés pour leur activité principale). Le chargement et déchargement des marchandises, le portage des bagages à l'abord des gares, procurent aussi des opportunités d'emplois appréciables aux sans-abri. Le transport des passagers par cyclo-rickshaw Tricycle servant au transport des passagers et des marchandises dans la ville..]]dans ces quartiers à la fois densément peuplés et aux multiples commerces, avec deux gares ferroviaires et une gare routière voisine, attire une autre proportion importante de sans-logis pouvant ainsi travailler à leur compte ( $20 \%$ des enquêtés pour 
leur activité principale). Au total, le secteur des transports absorbe la majorité des travailleurs sans-abri basés dans la vieille ville (52\% de l'échantillon pour l'activité principale). Le poids de ce secteur apparait comme la caractéristique majeure de la structure d'emploi des sans-logis par rapport à la population active de l'ensemble de la capitale, ou même de la seule population active résidant dans old Delhi : le secteur des transports et communications n'emploie que 8 à $9 \%$ des actifs correspondants ${ }^{35}$. Une autre spécificité d'old Delhi réside dans la présence de nombreux marchés du travail au strict sens néoclassique du terme -qui se tiennent à différents points de la vieille ville. Ces marchés sont souvent spécialisés dans le recrutement de certaines catégories de travailleurs : ouvriers du bâtiment ; ou encore cuisiniers, serveurs et autres emplois associés aux services de traiteurs et à l'organisation de grandes réceptions nécessitant le montage de vastes structures temporaires en toile destinées à accueillir de très nombreux invités. A Delhi, ce dernier type d'activité, liée plus particulièrement à l'organisation des réceptions de mariage, mobilise une main d'œuvre abondante, sur une base saisonnière, avec des pics correspondant aux périodes les plus propices à la célébration des unions. Les sans-logis s'embauchent volontiers dans ce secteur, en particulier pour une activité complémentaire sur une base saisonnière ou temporaire : ainsi la catégorie des serveurs, cuisiniers et autres aides ${ }^{36}$ comptent pour $22 \%$ des emplois reportés en activité principale par les sans-logis enquêtés, et pour $56 \%$ des emplois secondaires ${ }^{37}$. En revanche, les occupations marginales que l'on associe souvent à la condition des pauvres en ville, comme la mendicité ou le ramassage des papiers, plastiques, ferrailles, usagés, etc., en vue de leur recyclage ne concernent qu'un petit groupe minoritaire des sans-logis enquêtés à old Delhi ( $1 \%$ et $2 \%$ respectivement), la collecte des déchets étant surtout le fait d'enfants et d'adolescents. Forte variance des revenus et seuil de pauvreté Du point de vue de leurs revenus, les sans-logis constituent un segment très hétérogène de la force de travail urbaine : le revenu moyen (sur les 12 derniers mois précédant l'enquête de 1996) varie de 300 roupies par mois (cas d'un enfant aidant à servir le thé dans une échoppe) à 4500 (cas d'un adulte engagé dans diverses occupations illégales combinées à d'autres activités légales), avec $60 \%$ des sans-logis interrogés gagnant entre 1000 et 2000 roupies par mois pour leur seule activité principale. Les variations de revenu d'un individu à l'autre pour l'exercice d'une même activité sont également très fortes. Néanmoins, tous les sans-logis interrogés dans Old Delhi (à l'exception d'un cas) sont en mesure de générer des revenus les plaçant dans le groupe des bas revenus, certes, mais au dessus du seuil de pauvreté, fixé à 310 roupies par tête et par mois, et déterminé comme le niveau minimum de dépenses requis pour assurer une ration alimentaire journalière de 2100 calories par personne dans les zones urbaines, aux prix de 1995-96. Cette conclusion reste valable après retenue des envois d'argent à la famille à l'extérieur de Delhi. Proportionnellement à leur niveau de revenu, la capacité d'épargne de la majorité des sans-logis est en effet loin d'être négligeable. Si les revenus générés par les sans-logis enquêtés dans old Delhi leur permettent d'assurer leurs besoins nutritionnels minima, les plaçant ainsi au-dessus de la ligne de pauvreté, d'autres besoins essentiels, en particulier un logement décent, des vêtements, sans parler de l'accès aux soins de santé, autant d'aspects à prendre également en compte pour une approche plus compréhensive de la pauvreté, ne sont pas nécessairement couverts. La privation de logement est évidente pendant le séjour à Delhi, quant aux besoins vestimentaires, la tenue de certains sans-abri témoigne d'elle-même des besoins non satisfaits. Ainsi plusieurs des enquêtés ont reconnu n'avoir qu'une seule tenue, destinée à être portée 
jusqu'à devenir hors d'usage et nécessiter son remplacement. Dans ce cas toutefois le manque de pouvoir d'achat n'est pas le seul facteur explicatif, l'absence d'un lieu sûr pour pouvoir garder leurs effets personnels et leurs économies est un autre problème majeur auquel doivent faire face les sans-abri. Insécurité de l'emploi et irrégularité des revenus La préoccupation essentielle des travailleurs sans-abri est l'insécurité de l'emploi et l'incertitude quant au travail à venir. Se rendre quotidiennement ou périodiquement sur un marché du travail pour se faire embaucher par un recruteur, ou sur les marchés de gros et dans les rues du bazar pour trouver un travail à la tâche, est le sort commun des ouvriers du bâtiment, des serveurs dans la restauration, des portefaix, des tireurs de charrettes à bras, et autres manœuvres et travailleurs précaires. Parmi les sans-logis au statut d'employé, rares sont ceux qui ont un emploi salarié régulier. Quant aux travailleurs indépendants, comme les tireurs de charrettes à bras ou les tireurs de cyclo-rickshaw, ils doivent chaque jour payer la location de leur véhicule, sans aucune garantie quant aux nombres de courses à assurer. La situation des vendeurs de rue, des mécaniciens installés sur les trottoirs, et autres travailleurs indépendants du secteur informel, est similaire du point de vue de la précarité et de l'irrégularité des revenus. La flexibilité dans les types d'emplois occupés et la combinaison de plusieurs activités apparaissent comme une réponse au risque de chômage et à l'irrégularité du travail. Ainsi, près d'un tiers des sans-logis interrogés ont exercé plus d'une activité au cours des 12 derniers mois passés à Delhi, en général deux. Ces emplois différents sont souvent alternés en fonction des fluctuations des différents marchés du travail, en particulier selon un schéma saisonnier; plusieurs emplois peuvent également être occupés simultanément tout au long de l'année ${ }^{38}$. Si, en fin de compte, la plupart des sans-logis enquêtés dans old Delhi n'ont pas été affectés par le chômage au cours de l'année passée, et si, parmi ceux affectés, très peu ont déclaré des périodes d'inactivité de durée conséquente, l'insécurité de l'emploi et en conséquence l'absence de garantie d'un revenu régulier restent les traits les plus significatifs de la condition économique de cette population, et un aspect crucial pour comprendre leur situation de sans-abri (comme il sera développé dans la section suivante). Biens détenus dans la localité d'origine et projets d'investissement Pour compléter ce portrait mitigé du profil économique des sans-abri dans old Delhi et apprécier correctement leur condition, il est nécessaire de dépasser le cadre limité du séjour en ville, et de replacer les migrants dans leur contexte familial, en prenant en compte la situation économique au lieu d'origine et en envisageant la perspective d'un retour au village. Les personnes sans-logis à Delhi ne sont pas nécessairement issues des familles rurales les plus démunies. La plupart des personnes interrogées pour les entretiens approfondis déclarent avoir une maison familiale dans leur localité d'origine, et la majorité d'entre elles des terres agricoles - certes de petite superficie ${ }^{39}$. Pour ceux des migrants sanslogis qui ont maintenu des relations avec leur famille, soit la majorité d'entre eux, ces avoirs familiaux au lieu d'origine représentent aussi une sécurité, dans la mesure où cela implique en général des droits sur la terre ou autres propriétés. Inversement, la protection des droits sur les propriétés familiales agit comme un encouragement pour les migrants à maintenir des relations avec leur milieu d'origine et à l'envoi d'argent à leur famille. Comme couramment observé dans le processus de migration des campagnes vers les villes dans les pays en développement, l'accès à la terre et aux autres avoirs peut être utilisé par la famille et la communauté villageoise comme un levier pour contrôler les migrants, de manière à bénéficier des retours économiques de leur emploi en ville ${ }^{40}$. Parmi les migrants qui souhaitent retourner un jour dans leur 
village ou ville natale (soit la majorité des sans-logis interrogés), les projets d'investissements futurs sont fréquents, en particulier pour y ouvrir une épicerie générale, ou un autre type de commerce, pour acheter davantage de terres agricoles et plus généralement pour investir dans l'agriculture. Pour réaliser leurs projets d'investissement, les migrants sans-logis ont l'intention de mobiliser leur propre épargne, complétée si nécessaire par des contributions familiales. Certains de ces projets d'investissement ne verront peut-être jamais le jour, d'autres semblent viables étant donné la capacité d'épargne des travailleurs migrants concernés. Cela montre un degré appréciable de dynamisme économique parmi certains migrants sans-logis; on peut également y lire un calcul économique entraînant un sacrifice temporaire en termes de conditions de logement en ville, afin d'améliorer la situation économique au lieu d'origine. Quelle notion de pauvreté ? Cette analyse du profil économique des sansabri basés à old Delhi remet tout d'abord en question la marginalité économique de cette population : les sans-abri ne sont pas des indigents, ils participent pleinement à la force de travail urbaine, et les revenus qu'ils génèrent leur assurent plus qu'un minimum vital. De par leurs conditions d'emploi et de vie en ville, ils restent toutefois des travailleurs très vulnérables. La très grande variété des situations rencontrées montre également que les sans-abri sont loin de constituer une catégorie unique et homogène de pauvres en ville, pas plus qu'ils ne sont nécessairement les plus pauvres des pauvres urbains. Le contexte familial de la migration en ville des sans-abri permet finalement de souligner la relativité de la situation de pauvreté : relativité par rapport à l'espace de vie du migrant, et par rapport au temps de sa biographie. Ainsi, vivre dans une certaine situation de dénuement, sans abri, en ville, ne signifie pas nécessairement une absence de biens au lieu d'origine. Par ailleurs, le sacrifice d'un logement pendant le séjour en ville peut s'inscrire dans un projet d'amélioration des conditions de vie future de la famille au village natal, - c'est cette perspective que nous nous proposons d'explorer plus avant dans la section suivante. Exclusion du logement et stratégies économiques et résidentielles adaptatives En analysant les pratiques résidentielles et les stratégies économiques des sans-logis, nous tenterons dans cette section de discerner les éléments de contrainte et les éléments de choix, et d'en comprendre l'articulation : dans quelle mesure la situation de sans-abri est-elle la conséquence d'un processus d'exclusion du marché du logement urbain? et dans quelle mesure correspond-elle également à une stratégie résidentielle destinée à améliorer la condition économique des migrants et/ou de leur famille? Une autre question concerne la nature temporaire ou permanente de la situation de sans-abri : s'agit-il d'une étape transitoire précédant une meilleure insertion dans le parc des logements urbains ? ou bien cette situation correspond-elle à un mode de vie permanent, ou du moins pour la totalité du séjour à Delhi ? Des contraintes financières très pesantes mais relatives Dans des conditions économiques telles que celles décrites précédemment, les contraintes financières et l'incertitude des revenus représentent indéniablement un obstacle majeur pour l'accès à un logement. Ce facteur doit cependant être considéré dans une perspective à long terme et en relation avec les autres facteurs explicatifs intervenant, qui forment un système dans lequel des éléments de choix sont souvent présents. Par exemple, les contraintes financières sont probablement plus sévères au stade initial de la vie de sans-abri, en particulier quand cette situation correspond à l'arrivée à Delhi, et que le migrant doit s'insérer sur le marché du travail. Ensuite, les contraintes financières peuvent résulter non pas tant du niveau moyen de revenu, mais davantage de l'absence de garantie de revenu régulier. Cet élément d'incertitude 
empêche de nombreux sans-abri aux emplois précaires d'envisager la location d'un logement même s'ils disposent du potentiel financier correspondant, parce qu'un loyer engage des dépenses fixes non ajustables au revenu effectif. A l'inverse, les dépenses pour l'entrée dans un asile de nuit ou la location d'une couette, sont encourues sur une base journalière et peuvent en conséquence être facilement ajustées en fonction du revenu de la journée. Enfin, certains sans-logis qui disposeraient d'une capacité d'épargne suffisante pour une location (seul ou en la partageant), donnent la priorité aux envois d'argent à leur famille ou à l'épargne à long terme pour des projets d'investissements futurs dans la localité d'origine. En d'autres termes, la préférence est donnée aux conditions de vie de la famille au lieu d'origine sur les conditions de vie du migrant à Delhi, et au futur sur le présent. Dans ce cas, on ne peut pas dire qu'il y ait des contraintes financières absolues empêchant le migrant sans-abri de louer une pièce, mais plutôt des contraintes relatives, résultant de ses propres choix et priorités. Stratégies de rapprochement du lieu du travail La proximité entre le lieu de couchage et le lieu de travail s'avère un autre élément fondamental pour comprendre la situation de sans-abri de nombreux travailleurs migrants d'old Delhi et le choix de leur lieu de couchage. Une meilleure proximité du lieu du travail ou de la source d'emplois potentiels est une des raisons reportées explicitement par certains enquêtés pour expliquer leur arrivée initiale sur le trottoir ou dans un asile de nuit, et plus souvent pour expliquer le choix d'un emplacement spécifique pour la nuit. Les faits sont encore plus révélateurs que ces réponses explicites. Ainsi, une très forte proportion des personnes sans-logis interrogées dans old Delhi travaille dans la vieille ville même (pour l'activité principale $78 \%$ de l'échantillon), ou dans des quartiers adjacents. Si l'on s'en tient à l'activité principale, $80 \%$ des actifs sans-logis enquêtés se déplacent à pied à leur lieu de travail, le temps de transport moyen est de 16 minutes seulement par trajet, et pour $57 \%$ d'entre eux dix minutes ou moins. Le coût de transport quotidien au lieu de travail est en conséquence réduit à zéro pour la plupart des sans-logis. Un emplacement près du lieu de travail ou d'embauche permet ainsi aux sans-logis de minimiser - ou même supprimer - leurs dépenses de transport. Il permet aussi d'éviter la fatigue de navettes quotidiennes et d'assurer un repos adéquat, autre facteur vital pour les travailleurs manuels engagés dans des activités exigeant un effort physique intense. En outre, pour les journaliers, une plus grande proximité entre le lieu de couchage et le lieu d'embauche quotidienne accroît leur probabilité de trouver du travail. La mobilité résidentielle des sans-logis dans l'agglomération même de Delhi permet de souligner à nouveau l'importance des stratégies de rapprochement du lieu de travail. Les changements de lieux de couchage sont fréquemment observés au cours de l'année, selon la saison et les conditions climatiques d'une part, mais aussi suivant la mobilité professionnelle. Dans ce cas, les travailleurs sans-logis ajustent leur localisation résidentielle en fonction des opportunités d'emplois et de la localisation de ces derniers. La situation de sans-abri : étape transitoire ou mode d'insertion urbaine durable? La situation de sans-abri correspond-elle à une étape dans un processus d'insertion en ville ou se pérennise-t-elle ? Cette question permet d'aborder une autre facette de la place des sans-abri dans la ville. Il existe une proportion notable de sanslogis qui vivent dans cette condition depuis dix ans ou plus ( $16 \%$ de l'échantillon) - ce qui n'exclut pas toutefois des séjours réguliers dans la localité d'origine. Pour cette catégorie de sans-logis, en particulier pour ceux qui, en outre, fréquentent le même asile de nuit, restent sur le même coin de trottoir, ou répètent chaque année le même modèle saisonnier de changement de place de couchage depuis les dix ou même quinze 
dernières années, la condition de sans-abri semble être devenue un mode de vie permanent à Delhi, ou du moins pour la durée de leur vie active, avant le retour définitif au lieu d'origine. Le désir de nombreux sans-logis d'accéder à un logement décent à Delhi est susceptible de rester le plus souvent un vœu abstrait, étant donné les conditions - très contraignantes - nécessaires à la réalisation d'un tel projet : trouver une location bon marché pas trop éloignée du lieu de travail, et obtenir un revenu régulier et un emploi sûr comme exigence préalable à tout changement résidentiel. Les arguments de ceux qui déclarent ne pas vouloir changer pour un meilleur logement, et qui forment un groupe non négligeable, sont aussi révélateurs pour apprécier le caractère temporaire ou pérenne de la situation de sans-abri. Ceux qui ne veulent rien dépenser pour un logement ne sont pas nécessairement ceux qui n'ont aucune capacité d'épargne et donc aucun potentiel financier. Dans ce cas, deux types de raisons sont mis en avant pour expliquer l'absence de volonté de payer pour accéder à un logement :

- certains sans-logis n'ont pas l'intention de rester à Delhi pour une longue période, et trouveraient donc illogique et inutile de prendre une location ;

- d'autres donnent clairement la priorité à la maximisation de leur épargne, en particulier pour pouvoir envoyer de l'argent à la famille au «village », et ils essayent donc de minimiser leurs dépenses de logement et de transport, ou même de réduire ces dépenses à zéro. Restant seuls à Delhi, sans leur famille, certains migrants ne perçoivent pas un logement décent comme une nécessité pour eux. En fait, la plupart des sans-logis interviewés n'ont pas pour projet de s'installer définitivement à Delhi, mais de retourner un jour dans leur localité d'origine, ou bien d'émigrer vers une autre ville. Ainsi, ils perçoivent leur séjour à Delhi comme limité dans le temps (même si cette situation transitoire peut éventuellement se prolonger pour la durée de leur vie active), et en conséquence un logement n'est pas perçu comme un besoin prioritaire. Stratégies économiques ou tactiques de survie? En résumé, les pratiques résidentielles de la majorité des sans-logis révèlent des éléments de rationalité économique qui visent à maximiser l'épargne et les envois d'argent à la famille dans la localité d'origine, par la minimisation des dépenses de logement et de transport. Quand la logique de la situation de sans-abri à Delhi est une part intégrale des stratégies familiales enracinées au lieu d'origine, avec priorité donnée à la condition économique de la famille au village aux dépens des conditions de vie du migrant en ville, la situation de sans-abri du migrant a de fortes chances de se prolonger pendant toute la durée de son séjour dans la capitale. La part des éléments de choix rationnel dans les pratiques résidentielles des sans-logis a été mise en évidence dans d'autres études ${ }^{41}$. Ces travaux soulignent également l'importance des logiques de rapprochement du lieu de travail ou de la source d'embauche potentielle. Ce facteur est crucial non seulement pour comprendre les pratiques résidentielles et les choix de localisation des sans-logis, mais plus généralement des pauvres en ville ${ }^{42}$. Ainsi l'échec de nombreuses tentatives de relogement des habitants des bidonvilles loin de leur habitat initial s'explique-t-il par un manque de considération pour les facilités d'accès physique aux sources d'emplois. La condition des sans-abri en ville, comme celle des habitants des bidonvilles, doit être considérée en relation avec leurs besoins et leurs priorités. Ceci est une condition préalable indispensable en vue de la formulation de politiques de logement appropriées ou de tout programme de lutte anti-pauvreté. Si en rester à l'image de pauvres exclus du logement et des services urbains de base ne permet pas de progresser dans la compréhension de la genèse et de la persistance de la situation de sans-abri, étant 
donné les contraintes financières drastiques qui limitent fortement le champ des choix possibles pour les sans-logis, peut-on vraiment conclure l'analyse de leur trajectoire et de leurs pratiques résidentielles et économiques en termes de mise en œuvre de stratégies économiques à proprement parler, ou ne s'agit-il pas plutôt de tactiques de survie, adaptées à leur environnement urbain? Partageant les propos d'Anne Gautman sur les stratégies de défense, il nous semble que «si la notion de stratégie suppose l'existence de choix, l'emprise de contraintes fortes suppose elle aussi le recours à des stratégies spécifiques, destinées précisément à desserrer ces contraintes " ${ }^{43}$. On ne peut cependant oblitérer l'effet de reconstruction et de rationalisation a posteriori des comportements, par le chercheur d'une part, mais aussi par les acteurs eux-mêmes : cette mise en perspective, en projet, pourrait permettre aux sans-logis de donner un sens à leur trajectoire, de mieux accepter leurs conditions de vie en ville, de mieux en justifier la dureté et les aspects dégradants, à leurs propres yeux, mais aussi aux yeux des autres, y compris de l'interviewer. La place des sans logis dans l'espace urbain et dans la société civile Les pratiques d'occupation de l'espace mises en œuvre par les sans-logis dans old Delhi prennent place dans un jeu de rapports de force mues par des intérêts divergents - l'espace urbain étant une ressource rare, son accès génère compétition et conflits. L'usage mixte du sol qui caractérise la vieille ville (avec des densités résidentielles extrêmement élevées combinées à de fortes concentrations d'activités économiques) a ainsi entraîné la confrontation de stratégies opposées de différents acteurs, institutionnels (les organismes gouvernementaux de planification) et privés (les commerçants, les entrepreneurs, les sans-abri). En dépit des tentatives de régulation des planificateurs et en opposition aux objectifs du schéma directeur ${ }^{44}$, la prolifération des établissements commerciaux et industriels dans le vieux centre ville au cours des dernières décennies a accéléré la détérioration du parc immobilier ancien et a par ailleurs attiré une population flottante de travailleurs migrants dont l'insertion résidentielle reste extrêmement précaire. Ce processus a favorisé le développement d'activités économiques et de pratiques résidentielles en marge de la légalité: l'occupation la nuit des trottoirs et autres espaces publics par les migrants sans-logis et par les entrepreneurs offrant des services de couchage à ces derniers. Bien qu'opérant sans autorisation officielle, ces petites entreprises informelles suppléent à l'insuffisante capacité des asiles de nuit ouverts par la municipalité. Cependant, loin d'être reconnue pour sa fonction d'utilité sociale par les pouvoirs publics, l'activité de ces petits entrepreneurs provoquent plutôt des interférences avec la police: des menaces d'éviction pèsent ainsi constamment sur les entrepreneurs (assorties éventuellement de la confiscation de leur matériel de couchage) et sur les sans-abri dormant dans les espaces publics. De telles mesures sont exécutées de manière sporadique, et plus systématiquement à la veille de certaines manifestations publiques d'envergure (défilé à l'occasion de fêtes nationales par exemple), ou encore en période électorale " sensible ». Pour minimiser les risques d'éviction aléatoire à un échelon local, des potsde-vin sont régulièrement versés par les entrepreneurs aux agents de police contrôlant leur quartier. Quant aux opérations de "nettoyage » des rues décidées à plus haut niveau, elles traduisent l'opinion négative des autorités policières à l'égard des sansabri, et contribuent à la stigmatisation et à la criminalisation de cette population. Bien insérés dans le marché du travail local, rouage indéniable du dynamisme économique de la vieille ville, les sans-abri apparaissent pourtant d'abord comme des délinquants en puissance aux yeux des autorités policières, et les trottoirs et leurs occupants comme des repères potentiels de criminels et terroristes. En témoignent, par exemple, 
les opérations d'éviction et de ratissage policier des aires de couchage en plein air localisées dans la vieille ville suite aux attentats à l'explosif dans cette partie de la capitale. L'équation "classes laborieuses - classes dangereuses" semble toujours d'actualité à l'encontre des sans-abri. Lorsque la loi et le système judiciaire interviennent pour réglementer l'usage de l'espace urbain et résoudre les conflits dans lesquels se trouvent pris les sans-logis et les occupants des trottoirs, un parti-pris antipauvres est à nouveau prévalent. ${ }^{45}$. C'est ainsi que les textes de $10 \mathrm{i}^{46}$ confèrent des pouvoirs très étendus aux autorités municipales et de police pour appréhender les sans-abri sur la base de notions assez subjectives de "nuisance publique", "d'obstruction" de l'espace public et "d'intrusion", ou encore dans le cadre des mesures de prévention de la mendicité et du vagabondage. Et c'est ainsi que certains travailleurs migrants sans-abri se retrouvent enfermés dans des asiles - de fait des centres de détention - pour indigents, privés de leur liberté parfois pour plusieurs mois. Les récits de certains sans-logis interviewés dans old Delhi, comme les rapports d'études spécifiques ${ }^{47}$, attestent de l'arbitraire des arrestations ainsi que des préjugés et jugements léonins dans l'enceinte du tribunal spécialisé dans les cas de mendicité (Beggars Court). Une affaire célèbre d'éviction collective dont le jugement a été porté à la Cour Suprême fournira une dernière illustration de la place faite aux habitants des rues dans une autre grande métropole indienne : il s'agit de l'éviction d'un ensemble de familles qui vivaient dans des structures précaires installées sur les trottoirs à Bombay $^{48}$. D'un côté, le jugement reconnaît l'importance absolument vitale pour ces familles pauvres d'un emplacement sur le trottoir, afin de rester le plus proche possible des lieux de travail, compte tenu de leur condition économique ne leur permettant pas d'assurer des dépenses de transport quotidien. En réponse à l'argument des pétitionnaires sur les droits fondamentaux des squatters, la Cour statue même que le droit aux moyens d'existence (dont l'accès, dans le cas présent, justifie l'installation sur le trottoir) est inclus dans le droit de vivre, droit fondamental garanti par la Constitution. Cependant, après ce constat de nécessité économique et cette déclaration sans précédent sur les droits fondamentaux, la Cour place la prévention des nuisances publiques dans la rue avant la pauvreté dans sa hiérarchie des priorités et, en conséquence, l'installation sur le trottoir de ces familles est jugée illégale ${ }^{49}$. Logique implacable de la prédominance de l'intérêt général sur les intérêts privés, l'intérêt des passants est ainsi jugé supérieur à celui des pauvres ayant élu domicile sur le trottoir afin de survivre en ville. Dans une section préliminaire, nous avons émis une mise en garde contre une assimilation abusive entre «sans-abri » et «sans domicile fixe »: le domicile de référence ou foyer permanent de la majorité des sans-abri enquêtés dans le centre historique de Delhi - des migrants qui maintiennent des liens avec leur milieu d'origine et leur famille -se trouve ailleurs qu'à Delhi, dans leur village ou ville natale où ils se rendent régulièrement. Pourtant, pendant tout leur séjour dans la capitale, quelle qu'en soit la durée, c'est bien l'absence d'une adresse de référence en ville qui oblitère le statut de citadin et de citoyen à part entière des sans-logis : sans adresse, pas de carte de rationnement qui leur donnerait accès aux magasins vendant des produits de base à des prix subventionnés et qui en outre sert en Inde de pièce d'identité et de preuve de résidence, et donc pas de droit de vote à Delhi. Restant intégrés - pour la majorité d'entre eux - dans des réseaux familiaux et forts de leurs attaches au milieu d'origine, tous insérés dans l'économie urbaine, les sans-logis d'old Delhi voient leur place dans l'espace de la ville et leur statut de citoyen remis en question par le pouvoir d'État à travers ses fonctions administratives, policières et judiciaires. 


\section{NOTES}

1. Cette étude s'intègre dans un programme de recherche plus large sur les mobilités spatiales des populations de Delhi (voir V. Dupont, «Les 'rurbains' de Delhi », Espace, Population, Société, $n^{\circ} 2-3,1997$, pp. 225-240), financé par l'Institut de recherche pour le Développement (ex ORSTOM, (département Sociétés, Urbanisation, Développement), avec des financements complémentaires du CNRS dans le cadre de l'Action Concertée en Sciences Sociales ORSTOM-CNRS et du PIR-Villes. Ce programme a été mené en Inde avec la collaboration et le soutien logistique du Centre de Sciences Humaines de Delhi (Ministère des Affaires Etrangères) et de l'Institute of Economic Growth (Delhi). L'article présenté ici s'appuie en grande partie sur la communication suivante : V. Dupont, D. Tingal, « Residential and Economic Practices of Pavement Dwellers in old Delhi », Delhi, Institute of Economic Growth, Working Paper Series, N ${ }^{\circ}$ E/186/97, 1997, $41 \mathrm{p}$.

2. Arora (R.K.), Chhibbber (A.K), « Chilled nights on pavements in Delhi. A socioeconomic study of the children staying in ran baseras in winter ». New Delhi, Delhi Development Authority, Socio-economic division, Slum and Jhuggi-Jhompri Department, mimeo, 1985, p. 1.

3. NIUA, Dimension of Urban poverty. A Situational Analysis, New Delhi, National Institute of Urban Affairs, Research Study Series n² 25, 1986, p. 90.

4. Gupta (D.B.), Kaul (S.), Pandey (R.), Housing and India's Urban Poor, New Delhi, HarAnand Publications, 1993, p. 29 (notre traduction).

5. Voir par exemple le dossier de la revue Sociétés Contemporaines : Les sans-domicile. Etats-Unis, France, n 30, avril 1998.

6. Dans la suite de ce texte le terme « Old Delhi » désignera uniquement cette partie de la capitale, c'est à dire la vieille ville délimitée par son enceinte fortifiée (voir section 3), alors que le terme « Delhi » se réfère à l'ensemble de l'agglomération urbaine 7. Jagannathan (N.V.), Halder (A.), «Income-housing Linkages. A case Study of Pavement dwellers in Calcutta ». Economic and Political Weekly, vol XXIII, $n^{\circ} 23$, June 4, 1988, p. 1175.

8. Voir par exemple : Alam (S. Manzoor), Alikhan (F.) (eds.), Poverty in Metropolitan Cities, New Delhi, Concept Publishing Company, 1987 ; Singh (A. M.), Souza (A. de), The Urban Poor, Slum and Pavement Dwellers in Major Cities of India, Delhi, Manohar, 1980 ; Souza (A. de) (ed.), The Indian City. Poverty, Ecology and Urban Development., Delhi, Manohar,1983.

9. Pour citer quelques références parmi les plus récentes : Nagpaul (H.), Modernisation and Urbanisation in India : Problems and Issues, Jaipur and New Delhi, Rawat Publications, 1996 ; Gupta (D.B.), Kaul (S.), Pandey (R.), Housing and India's Urban Poor, New Delhi, Har-Anand Publications, 1993 ; Jain (A.K.), 1996. The Indian Megacity and Economic Reform. Management Publishing Company, New Delhi ; Kundu (A.), In the Name of the Urban Poor. Access to Basic Amenities, New Delhi, Sage Publications, 1993 ; NIUA, Dimension of Urban poverty. A situational analysis, New Delhi, National Institute of Urban Affairs, Research Study Series n ${ }^{\circ} 25,1986$; NIUA, Profile of the Urban Poor : an Investigation into their Demographic, Economic and Shelter Characteristics, New Delhi, National Institute of Urban Affairs, Research Study Series n 40, 1989 ; Pernia (E.M.) (ed.), Urban Poverty in Asia. A Survey of Critical Issues, Hongkong, Oxford University 
Press, 1994 ; Suri (P.), Urban Poor. Their Housing Needs and Government Response, Delhi, Har-Anand Publications, 1994.

10. On peut toutefois citer (liste non exhaustive) pour Delhi : Arora (R.K.), Chhibbber (A.K), « Chilled nights on pavements in Delhi. A socio-economic study of the children staying in ran baseras in winter ", New Delhi, Delhi Development Authority, Socioeconomic division, Slum and Jhuggi-Jhompri Department, mimeo, 1985 ; Bharat Sevak Samaj, " A roof over the head », Delhi, Delhi School of Social Work, 1964 ; DDA, "Survey of pavement dwellers in Old Delhi », New Delhi, Delhi Development Authority, Slum and Jhuggi-Jhompri Department, mimeo, 1989 ; Kuruvilla (J.), « Pavement dwelling in metropolitan cities. Case study Delhi », Thesis, New Delhi, School of Planning and Architecture, Dept. of Housing, mimeo, 1990-91.

11. La principale publication est une étude spéciale sur les sans-abri menée au cours des opérations du recensement de 1971 (Gandotra (S. R.), Houseless in Delhi, Census of India 1971, Series 27, Delhi, Part X (a), Special study, Delhi, Government of India, 1976). Les autres références correspondent à des études non publiées et mal diffusées : le rapport pionnier en la matière du Bharat Sevak Samaj (op. cit., 1964), une organisation non gouvernementale dédiée à la cause des pauvres ; une enquête effectuée en 1989 par le département des bidonvilles de Delhi - mais le rapport, non publié, est à présent introuvable y compris dans les archives de cette administration (Arora, Chhibber, op. cit., 1985) ; et une thèse de maîtrise en architecture et urbanisme reposant sur des enquêtes de terrain effectuées en 1990 (Kuruvilla, op. cit., 1990-91).

12. fournies par le Slum and Jhuggy-Jhompri Department de la Delhi Development Authority.

13. Jagannathan, Hadler, op. cit., 1988, p. 1175.

14. Ramanchandran, op. cit., 1972 ; Kuruvilla, op. cit., 1990-91 ; SPARC, 1985, op. cit. 15. Census of India, Instructions to enumerators for filling up the household schedule and individual slip, New Delhi, Ministry of Home Affairs, Office of the registrar General \& Census Commissioner for India, 1991, p. 64.

16. Bienveniste (C.), « Pour une meilleure connaissance des sans-abri et de l'exclusion du logement », Actualités du Conseil National de l'Information Statistique, $\mathrm{n}^{\circ} 17$, mai 1996, p. 1.

17. Wright (J. D.), Rubin (B.), op. cit., 1998.

18. Les entretiens approfondis ont été conduits par l'auteur avec la collaboration de Dhananjay Tingal. Pour plus de détails sur la méthodologie des enquêtes, voir : Dupont (V.), « City history - life histories : changing equations. Migration surveys and biographical data collection in Delhi », New Delhi, Centre de Sciences Humaines, Contributions CSH 97/7, 1997.

19. Voir Brush (J.), « Recent changes in ecological patterns of metropolitan Bombay and Delhi », in Tewari (V. K.), Weistein (J. A.), Rao (V. L. S. P.) (eds), Indian Cities. Ecological Perspectives, New-Delhi, Concept, 1986, pp. 121-149.

20. DDA, Zonal Plan Walled City. Draft, New Delhi, Delhi Development Authority, 1993

21. Merha (A. K.), The Politics of Urban Redevelopment. A Study of Old Delhi, New Delhi, Sage Publications, 1991, p. 50.

22. Voir Indian Human Settlement Programme, Renewal of historical housing stock in Old Delhi, New Delhi, Human Settlement Management Institute, HSMI Studies 1, 1988.

23. Voir aussi : TCPO, Seminar on Redevelopment of Shahjahanabad : the Walled City of Delhi (New Delhi, 31st Jan.- 1st Feb. 1995), Report and Selected Papers. Resume of the 
Seminar, New Delhi, Government of India, Ministry of Works and Housing, Town and Country Planning Organisation, 1975.

24. Voir la conclusion.

25. Dans les zones de couchage à l'extérieur situées dans l'enceinte de la vieille ville, quelques cas seulement de femmes et de familles sans-logis (moins d'une dizaine) ont pu être repérés pendant la période d'observation, et leur présence était en outre limitée à un ou deux emplacements. Par ailleurs, l'entrée dans les asiles de nuit municipaux est strictement réservée aux hommes. En conséquence, l'échantillon enquêté est composé exclusivement de sans-logis de sexe masculin (adultes ou enfants. 26. Au recensement de 1991, les données sur l'alphabétisation se rapportent à la population âgée de 7 ans et plus.

27. Dans l'ensemble de l'échantillon enquêté, des raisons familiales de ce type ont été données par $24 \%$ des sans-logis comme raison principale de la migration vers Delhi. En outre, environ un tiers des 36 personnes sélectionnées pour les interviews approfondies ont rapporté ce type de raison au cours de leurs étapes migratoire.

28. Dupont (V.), « Les rurbains de Delhi », Espaces, Populations et Sociétés, Numéro spécial sur Les Populations du Monde Indien, n² 2/3, 1997, pp. 225-240.

29. Voir par exemple Banerjee (B.), Rural to Urban Migration and the Urban Labour Market (A case study of Delhi), Delhi, Himalaya Publishing House, 1986.

30. Les activités prises en compte comprennent toutes les activités exercées à Delhi pendant les 12 derniers mois précédant l'enquête. Les activités exercées pendant la période de référence mais dans un autre lieu que Delhi (en particulier dans le village d'origine ou au lieu de résidence antérieur) sont exclues de ce tableau. (ne travaillent pas $=5$ cas). Source : Enquêtes ORSTOM-IEG - 1996.

31. Source : (1) Enquêtes ORSTOM-IEG - 1996. Echantillon de 243 actifs ayant un emploi, classifiés selon leur activité principale (ne travaillent pas $=5$ cas) (2) \& (3) : Census of India 1991, Delhi, Primary Census Abstract.

32. Contrairement aux conventions appliquées dans l'élaboration des statistiques officielles en Inde (recensements, enquêtes nationales), dans cette étude les mendiants sont inclus dans la catégorie des actifs occupés ; ils ne comptent d'ailleurs que pour moins de $1 \%$ des travailleurs sans-logis enquêtés dans la vieille ville. .

33. Arora, Chhibber, op. cit., 1985, p. 5. .

34. Cf. tableau 1..

35. Cf. tableau 2..

36. Dans le contexte de l'enquête auprès des sans-logis, cette catégorie professionnelle correspond essentiellement aux journaliers employés par les services de traiteurs et pour l'organisation de réceptions, mais elle inclut également les cuisiniers, serveurs et autres aides embauchés dans les restaurants et stands de thé.

37. Cf. tableau 1.

38. Cf. tableau 1.

39. Certains enquêtés sont même issus de familles aisées, leur migration dans ce cas a plus de chances de résulter de disputes familiales que de facteurs économiques..

40. Guilmoto (C. Z.), Sandron (F.), « Approche institutionnelle de la migration dans les pays en développement », Paper presented to the XXIIIth General Population Conference, IUSSP, Beijing (Chine), 11-17 October 1997.

41. Par exemple, en conclusion d'une enquête auprès des sans-logis à Delhi, Kuruvilla estime que : «Le choix du trottoir vise principalement à réduire les dépenses de logement, à rester à proximité du lieu de travail, (....) et à maximiser les économies 
envoyées à la famille. Ainsi rester sur le trottoir devient une décision délibérée et rationnelle » (Kuruvilla, op. cit., 1991-92, pp. 85-86, notre traduction). Jagannathan et Halder, dans leur étude des sans-abri à Calcutta, concluent également : « Les sans-logis engagés dans des activités non marginales ont choisi ce style de vie pour protéger leur accès aux sources de revenus. En outre (...) une proportion substantielle sont des migrants temporaires, qui envoient leurs économies à leur famille au village ». Plus loin : « La majorité des personnes vivant sur le trottoir vivent ainsi sans abri suivant une décision délibérée et rationnelle » (Jagannathan et Halder, op. cit., 1988, p. 1177, notre traduction).

42. Voir par exemple : Bharat Sevak Samaj, Slums of Old Delhi, Delhi, Atma Ram \& Sons, 1958, p. 107 ; Gupta, Kaul, Pandey, op. cit., 1993, p. 86 ; Suri, op. cit., 1994, p. 273 ;

Kundu, op. cit., 1993, p. 65.

43. Gautman (A.), «Stratégies résidentielles, stratégies de recherche », in : Stratégies résidentielles. Séminaire organisé par C. Bonvalet et A. M. Fribourg (Paris, 1988), Paris, INED, Plan Construction et Architecte, MELTM - 1990, p. 30.

44. Voir DDA, Master Plan for Delhi. Perspective 2001, New Delhi, Delhi Development Authority, 1990.

45. Pour une analyse systématique des litiges d'intérêt public autour des enjeux de l'espace urbain en Inde, voir : Ahuja (S.), People, Law and Justice. Casebook on Public Interest Litigation, New Delhi, Orient Longman, 1997, chap. 8 ; pour une approche plus générale des rapports de la loi à la pauvreté en Inde, voir Baxi (U.) (ed.), Law and Poverty. Critical Essays, Bombay, N.M. Tripathi, 1988.

46. Voir par exemple The Beggary Prevention Act, et dans le cas de Delhi en particulier : The Delhi Municipal Corporation Act, 1957 et The Delhi Police Act, 1978. 47. Voir Pande (B.B.), «Vagrants, Beggars and Status Offenders », in Baxi, op. cit., 1988, pp. 248-276.

48. Il s'agit d'un litige d'intérêt public connu sous le nom de « Olgas Tellis v. Bombay Municipal Corporation » (Supreme Court Cases, 1985) (3), pp. 555-590); voir aussi Ahuja, op. cit., 1997, chap. 8.

49. Ahuja, op. cit., 1997, p. 341.

INDEX

Index géographique : Inde

Mots-clés : économie politique, exclusion, pauvreté, villes/ sociologie urbaine 